\title{
Exciton dynamics studied via internal THz transitions
}

\author{
R. A. Kaindl ${ }^{1}$, D. Hägele ${ }^{1,2}$, M. A. Carnahan ${ }^{1}$, R. Lövenich ${ }^{1}$, and D. S. Chemla ${ }^{1}$ \\ ${ }^{1}$ Department of Physics, University of California at Berkeley and Materials Sciences Division, \\ E. O. Lawrence Berkeley National Laboratory, Berkeley, CA 94720, USA \\ ${ }^{2}$ Institut für Festkörperphysik, Universität Hannover, Appelstraße 2, 30167 Hannover, Germany
}

We employ a novel, ultrafast terahertz probe to investigate the dynamical interplay of optically-induced excitons and unbound electron-hole pairs in GaAs quantum wells. Resonant creation of heavy-hole excitons induces a new low-energy oscillator linked to transitions between the internal exciton degrees of freedom. The time-resolved terahertz optical conductivity is found to be a probe well suited for studies of fundamental processes such as formation, relaxation and ionization of excitons.

\section{Introduction}

Electron-hole $(e-h)$ gases in semiconductors represent a well-defined laboratory for investigating complex many-body interactions between large numbers of quasi-particles, phonons and photons [1-3]. The longrange Coulomb interaction between electrons and holes can stabilize the formation of excitons as hydrogen-like bound states with new physical properties. Intrinsically, charge-neutral excitons are electrically insulating due to the strongly correlated motion of electrons and holes, up to a frequency that equals the separation between their lowest internal states [4]. In contrast, unbound $e$-h pairs can form a conducting ionized gas. Dynamical transitions between these extreme phases are many-body effects that occur on short timescales. They are, however, difficult to measure since near-visible optical techniques typically employed, such as luminescence, are mostly sensitive to a subset of exciton states with vanishing center-of-mass momentum [5]. A new route to probing ultrafast exciton dynamics is discussed here. We employ transitions between internal states of excitons which, in analogy to atomic physics, are sensitive to higher center-of-mass momenta as well [6]. Due to small effective masses and large dielectric constants in typical semiconductors, the binding energy of excitons lies in the meV range, several orders of magnitude below the optical band gap. Internal transitions thus occur at terahertz $(\mathrm{THz})$ frequencies, where $1 \mathrm{THz}$ corresponds to $\approx 4 \mathrm{meV}$ photon energy [7,8]. Time-resolved $\mathrm{THz}$ experiments determine the full complexvalued dielectric response, thus significantly constraining theoretical models [9]. These arguments motivate using $\mathrm{THz}$ internal exciton transitions to probe exciton dynamics.

\section{Optical-Pump THz-Probe Experiments}

We investigate high-quality multiple-quantum wells grown by molecular beam epitaxy. They consist of ten 14-nm wide GaAs quantum wells separated by $10-\mathrm{nm}$ wide $\mathrm{Al}_{0.3} \mathrm{Ga}_{0.7} \mathrm{As}$ barriers [10]. To avoid effects from the substrate, the layers were attached to $\mathrm{MgO}$ and the substrate was removed. Ultrashort nearinfrared pump pulses used to excite either excitons or unbound electron-hole pairs are obtained from a $250-\mathrm{kHz}$ Ti:sapphire regenerative amplifier. Selective excitation is achieved by spectral narrowing to about $2 \mathrm{meV}$ bandwidth in a grating-based pulse shaper. Probe pulses of $\approx 500 \mathrm{fs}$ duration spanning the $0.5-3 \mathrm{THz}$ range are generated and detected in ZnTe crystals using optical rectification and electro-optic sampling, respectively. 


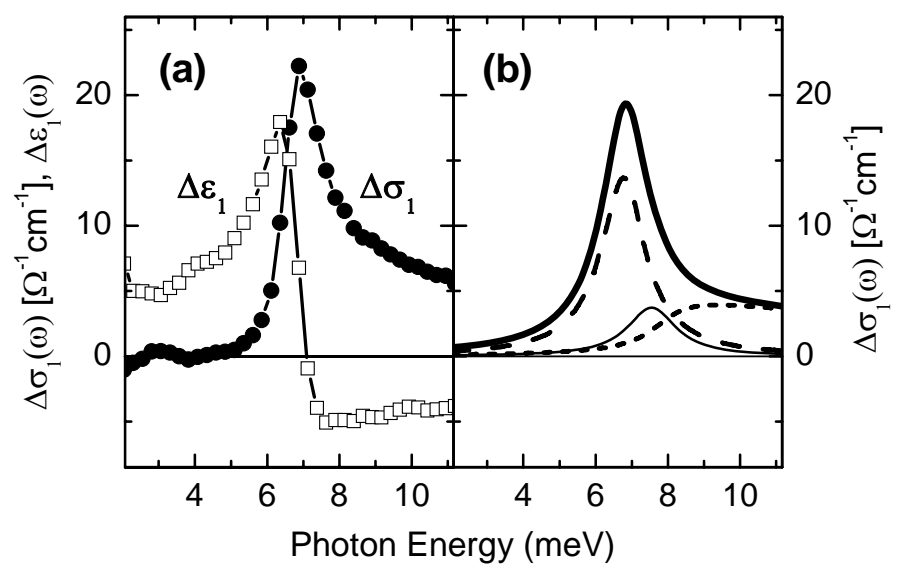

Fig. 1 (a) Change in THz conductivity $\Delta \sigma_{1}$ (dots), and in the dielectric function $\Delta \varepsilon_{1}$ (open circles), at time delay $\Delta \mathrm{t}=5 \mathrm{ps}$ after resonant excitation $(\mathrm{E}=1.540 \mathrm{eV})$ of $n \approx 1 \times 10^{10} \mathrm{~cm}^{-2} \mathrm{HH}$-excitons. The data were taken at lattice temperature $T_{\mathrm{L}}=6 \mathrm{~K}$. Lines are a guide to the eye. (b) simulated induced conductivity $\Delta \sigma_{1}$ (thick solid line), calculated by taking into account the dipole transitions originating from the $1 \mathrm{~s}$ exciton ground state. The absorption processes underlying this shape are $1 \mathrm{~s}-2 \mathrm{p}$ (dashed), $1 \mathrm{~s}-$ higher bound states (thin line) and 1s-continuum (short dashed line). For comparison, the peak height was scaled to the experimental data.

For each pump-probe delay $\Delta t$, we measure the THz field $E(t)$ transmitted through the unexcited sample and the pump-induced change $\Delta E(t)$. The transient change in the dielectric function is obtained from straightforward electrodynamical relations by including phase shifts in the multilayer structure [11]. The frequency-dependent optical conductivity $\sigma(\omega)=\sigma_{1}(\omega)+i \cdot \sigma_{2}(\omega)$ expresses the collective current response to the incident electric field. In the following, the response is analyzed in terms of both (i) its real part $\sigma_{1}(\omega)$ which yields a measure of absorptive processes and (ii) its dispersive imaginary part which is conveniently expressed via the real part of the dielectric function: $\sigma_{2}(\omega)=\omega / 4 \pi\left[1-\varepsilon_{1}(\omega)\right]$. The availability of both parts restricts theoretical modeling and clarifies the underlying physics.

\section{Internal exciton transitions}

At low lattice temperature $\mathrm{T}=6 \mathrm{~K}$ and after resonant excitation at the $\mathrm{HH}-1 \mathrm{~s}$ exciton line, a strong peak is observed in the $\mathrm{THz}$ conductivity around $\approx 7 \mathrm{meV}$ photon energy. After initial dephasing of coherent polarizations within a few ps [10] incoherent $1 \mathrm{~s}$ exciton populations remain. Internal exciton transitions occur as shown in Fig. 1(a) for an excitation density $\mathrm{n} \approx 10^{10} \mathrm{~cm}^{-2}$ and at $5 \mathrm{ps}$ time delay. The dispersive feature seen in $\Delta \varepsilon_{1}(\omega)$ corroborates the formation of this new far-infrared oscillator.

As presented in Fig 1(b), we have carried out calculations of such transitions using quasi-2D exciton wavefunctions [12]. The resulting shape (thick black line) is in good agreement with the experimentally observed shape in $\Delta \sigma_{1}(\omega)$, which is also true for $\Delta \varepsilon_{1}(\omega)$ although not explicitly shown. The absorption processes that underlie this asymmetric shape can be analyzed: the peak around $7 \mathrm{meV}$ is due to the $1 \mathrm{~s} \rightarrow 2 \mathrm{p}$ transition (dashed line), and its energy location is in good agreement with that from reported binding energies [13]. The higher-energy shoulder, in turn, is explained by transitions from 1s into higher bound states (thin line) and into the unbound $e$ - $h$ pair continuum (short dashed line).

At low lattice temperature, $T_{\mathrm{L}}=6 \mathrm{~K}$, the magnitude of the induced conductivity change decays with time while the peaked shape is preserved (not shown). Therefore, under these conditions the signals follow the population decay due to recombination. An elementary aspect concerns the vanishing of conductivity at the low-energy end of the spectrum as seen in Fig 1(a). This insulating nature of excitons is linked to the strongly correlated charge motion of its constituent electron and hole. 


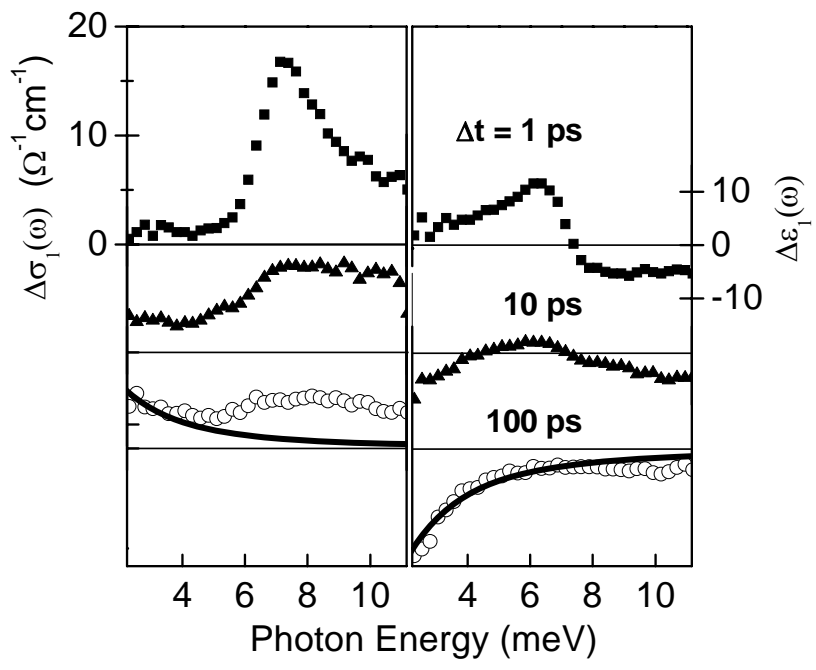

Fig. 2 Transient spectra after HH-1s resonant excitation of $n \approx 10^{10} \mathrm{~cm}^{-2} \mathrm{HH}$ excitons at elevated lattice temperature $T_{\mathrm{L}}=60 \mathrm{~K}$. All panels for different delay times are equally scaled in height like the $\Delta t=1 \mathrm{ps}$ curve. The thick solid line at $\Delta t=100 \mathrm{ps}$ is a Drude model calculation $\left(n \approx 1 \times 10^{10} \mathrm{~cm}^{-2}, 1 / \tau=0.5 \mathrm{THz}\right)$.

\section{Ionization dynamics}

A entirely different response is observed after resonant excitation of $\mathrm{HH}$ excitons at elevated lattice temperatures. The case of $T_{\mathrm{L}}=60 \mathrm{~K}$ is shown in Fig. 2. Initially, the experiment ( $\Delta t=1 \mathrm{ps}$ in Fig 2) is still approximated by the characteristic exciton peaked asymmetric lineshape, vanishing low-frequency conductivity, and oscillator-like dispersion. Additional broadening is observed already at $\Delta t=1 \mathrm{ps}$ which can be attributed to exciton dephasing through scattering with phonons. Within a few picoseconds, the peaked response in $\Delta \sigma_{1}(\omega)$ broadens to much larger values than any initial broadening. As low-frequency conductivity is acquired in $\Delta \sigma_{1}(\omega)$, the dielectric profile in $\Delta \varepsilon_{1}(\omega)$ changes into the all-negative Drude-like response. It is important to note that a simply broadened exciton peak would always show a positive $\Delta \varepsilon_{1}(\omega)$ for frequencies below its transition energy and thus cannot explain this behavior. The dynamics follows the ionization of the initially cold excitons via absorption of thermal phonons [1]. However, it is important that the THz conductivity in the ionized state is not wholly described by the Drude model (thick line, $\Delta \mathrm{t}=100 \mathrm{ps}$ ). Rather, it shows a strong trace of a broadened excitonic enhancement above $6 \mathrm{meV}$, which indicates the survival of such excitonic correlations.

\section{Conclusions}

We report on recent time-resolved experiments that study fundamental processes of exciton physics by probing internal exciton transitions at $\mathrm{THz}$ frequencies. In particular, resonant generation of excitons results in a low-energy response closely described by transitions between an exciton's internal degrees of freedom. Dynamical changes between bound and unbound $e-h$ pairs can be measured as is illustrated for the case of exciton ionization at $\mathrm{T}=60 \mathrm{~K}$. Changes in $\sigma_{1}(\omega)$ and $\varepsilon_{1}(\omega)$ are shown to be sensitive indicators for the transient state of the many-body $e-h$ gas. The dynamics of exciton formation is particularly interesting, results of which will be reported elsewhere [14]. The distinctively different nature of $\mathrm{THz}$ conductivity compared to interband probes renders it interesting for future exciton studies. 
Acknowledgements We thank J. Reno (Sandia Natl. Lab.) for growth of the quantum wells. This work was supported by the Director, Office of Science, Office of Basic Energy Sciences, Division of Materials Sciences, of the US Department of Energy under Contract No. DE-AC03-76SF00098, and through fellowships of the Deutsche Forschungsgemeinschaft and Alexander von Humboldt Foundation.

\section{References}

[1] J. Shah, Ultrafast Spectroscopy of Semiconductors and Semiconductor Nanostructures. Springer (1999).

[2] D. S. Chemla and J. Shah, Nature 411, 549 (2001).

[3] I. Perakis, Nature 417, 33 (2002).

[4] H. Haug, and S. Schmitt-Rink, J. Opt. Soc. Am. B2, 1135 (1985)

[5] some materials with large $e-p h$ coupling exhibit phonon replica in luminescence linked to excitons at nonvanishing momenta, see M. Umlauff et al. Phys Rev. B57, 1390 (1998).

[6] M. Kira, W. Hoyer, T. Stroucken, and S. W. Koch, Phys. Rev. Lett. 87, 176401 (2001).

[7] T. Timusk, Phys. Rev. B13, 3511 (1976).

[8] J. Černe et al., Phys. Rev. Lett. 77, 1131 (1996).

[9] R. Huber, F. Tauser, A. Brodscheim, M. Bichler, G. Abstreiter, and A. Leitenstorfer, Nature 414, 286 (2001).

[10] R. Lövenich, C. W. Lai, D. Hägele, D. S. Chemla, and W. Schäfer, Phys. Rev. B66, 045306 (2002).

[11] M. C. Nuss, and J. Orenstein, in: Millimeter and Submillimeter Wave Spectroscopy of Solids, edited by G. Grüner, Springer Verlag, Berlin (1998).

[12] H. Haug, and S. W. Koch, Quantum Theory of the Optical and Electronic Properties of Semiconductors World Scientific Publ., Singapore (1994).

[13] B. Gerlach, J. Wüsthoff, M. O. Dzero, and M. A. Smondyrev, Phys. Rev. B58, 10568 (1998).

[14] R. A. Kaindl et al. (to be published).

\section{DISCLAIMER}

This document was prepared as an account of work sponsored by the United States Government. While this document is believed to contain correct information, neither the United States Government nor any agency thereof, nor The Regents of the University of California, nor any of their employees, makes any warranty, express or implied, or assumes any legal responsibility for the accuracy, completeness, or usefulness of any information, apparatus, product, or process disclosed, or represents that its use would not infringe privately owned rights. Reference herein to any specific commercial product, process, or service by its trade name, trademark, manufacturer, or otherwise, does not necessarily constitute or imply its endorsement, recommendation, or favoring by the United States Government or any agency thereof, or The Regents of the University of California. The views and opinions of authors expressed herein do not necessarily state or reflect those of the United States Government or any agency thereof or The Regents of the University of California. 